\title{
Development of Nuclear Magnetic Resonance Pulse Sequences and Probes to Study Biomacromolecules
}

\author{
V. V. Krishnan, R. Maxwell
}

February 26, 2001

U.S. Department of Energy

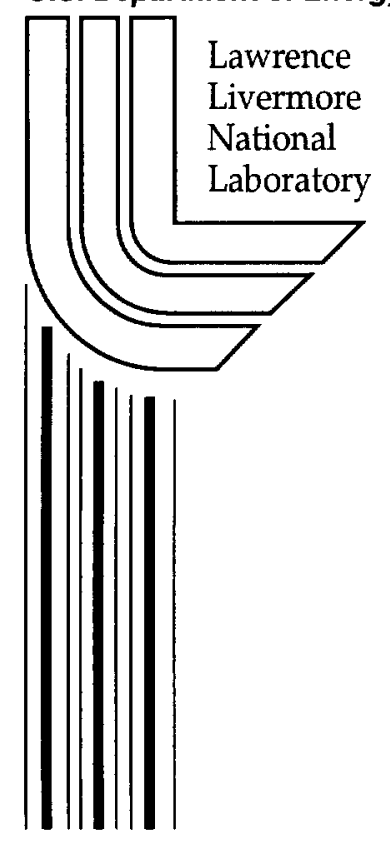




\section{DISCLAIMER}

This document was prepared as an account of work sponsored by an agency of the United States Government. Neither the United States Government nor the University of California nor any of their employees, makes any warranty, express or implied, or assumes any legal liability or responsibility for the accuracy, completeness, or usefulness of any information, apparatus, product, or process disclosed, or represents that its use would not infringe privately owned rights. Reference herein to any specific commercial product, process, or service by trade name, trademark, manufacturer, or otherwise, does not necessarily constitute or imply its endorsement, recommendation, or favoring by the United States Government or the University of California. The views and opinions of authors expressed herein do not necessarily state or reflect those of the United States Government or the University of California, and shall not be used for advertising or product endorsement purposes.

This work was performed under the auspices of the U. S. Department of Energy by the University of California, Lawrence Livermore National Laboratory under Contract No. W-7405-Eng-48.

This report has been reproduced directly from the best available copy.

Available electronically at http://www.doc.gov/bridge

Available for a processing fee to U.S. Department of Energy

And its contractors in paper from

U.S. Department of Energy

Office of Scientific and Technical Information P.O. Box 62

Oak Ridge, TN 37831-0062

Telephone: (865) 576-8401

Facsimile: (865) $576-5728$

E-mail: reports@adonis.osti.gov

Available for the sale to the public from

U.S. Department of Commerce

National Technical Information Service

5285 Port Royal Road

Springfield, VA 22161

Telephone: (800) 553-6847

Facsimile: (703) 605-6900

E-mail: orders@ntis.fedworld.gov

Online ordering: http://www.ntis.gov/ordering.htm

OR

Lawrence Livermore National Laboratory

Technical Information Department's Digital Library

http://www.llnl.gov/tid/Library.html 
Tracking Code: 99-LW-031

Principal Investigator: Monique Cosman, BBRP

Co-Investigators: V. V. Krishnan, BBRP and Robert Maxwell, C\&MS

Final Report: February 26, 2001

The determination of the three dimensional structures at high resolution of biomolecules, such as proteins and nucleic acids, enables us to understand their function at the molecular level. At the present time, there are only two methods available for determining such structures, nuclear magnetic resonance (NMR) spectroscopy and X-ray diffraction. Compared to well-established X-ray diffraction techniques, NMR methodology is relatively new and has many areas in which improvement can still be attained.

In this project, we focused on the development of new NMR probes and pulse sequences that were tailored to tackle specific problems that are not adequately addressed by current technology. Probes are the hardware that contain the radio frequency (RF) circuitry used to both excite and detect the NMR signals. Pulse sequences are composed of a series of RF pulses and delays, which are applied to the sample held within the magnetic field by the probe, so as to manipulate the nuclear spins. Typically, a probe is developed for a specific set of nuclei and types of experiments and the pulse sequences are then written to use the probe in an optimal manner. In addition, the inter-development of instrumentation and methods are determined by the specific biological question to be examined. Thus our efforts focused on addressing an area of importance in NMR Structural Biology namely more effective ways to use the phosphorus $\left({ }^{31} \mathrm{P}\right)$ nucleus. Phosphorus is a very important biological element that is strategically located in nucleic acids, where it imparts negative charge and flexibility to RNA and DNA. It is also a component of the cellular membrane and thus interacts with membrane proteins. It is used in mechanisms to signal, activate or deactivate enzymes; and participates in energy storage and release. However, the phosphorus nucleus exhibits certain properties, such as poor spectral dispersion, low sensitivity of detection, and fast relaxation, which limit its effective use in NMR studies of biomolecules. Our unique combination of expertise at LLNL allowed us to tackle each of the negative features of ${ }^{31} \mathrm{P}-\mathrm{NMR}$ in a three-pronged, concerted effort. The nature of our work necessitated an interdependent, multidisciplinary approach that required knowledge of spin physics (pulse sequences), engineering (probes), and structural biology (sample preparation and structure determination).

Central to our studies, is the preparation of suitable, biologically relevant samples that were needed not only for the testing of our new probes and pulse sequences, but also to overcome the poor spectral dispersion of ${ }^{31} \mathrm{P}$ in our NMR data. We synthesized DNA molecules that were modified by environmental carcinogens and food mutagens so that the phosphodiester backbone was severely distorted. Using these samples, we obtained 
order-of-magnitude better signal dispersions in the phosphorus spectra. The sample preparation aspect of this project also included the preparation of a repair protein, BRCT (breast cancer C- terminus) domain of ligase III) and a membrane protein, (myelin oligodendrocyte glycoprotein (MOG)), which is an important self-antigen implicated in human multiple sclerosis. The preparation of these samples resulted in the recent submittal of one paper to Proceedings National Academy of Science, and another in press in Protein Expression and Purification. Another paper on the structure and dynamics of the BRCT domain of ligase III $\alpha$ will be submitted soon to Biochemistry. Much of our work involving these samples have also been presented at several national meetings: "Chemical shift perturbation studies of the DNA repair protein XPA complexed with benzo[a]pyrene-DNA adducts" and "Solution Structure of the ligase III $\alpha$ BRCT domain" presented at the $41^{\text {st }}$ Experimental NMR Conference, Asilomar, CA, April 9-14, 2000; "Structural Plasticity of a protein antigen implicated in the immunopathogenesis of multiple sclerosis" presented at the American Neurological Association Meeting, Seattle WA, October 6-10, 1999; "Investigation of the secondary structural changes in rMOG(1125) by circular dichroism" presented at the American Chemical Society Regional Meeting, New Orleans, December, 2000, and "Solution structure of the 2-amino-1methyl-6-phenylimidazo[4,5-b]pyridine C8-deoxyguanosine adduct opposite $\mathrm{dC}$ in a duplex DNA" and "Biophysical characterization studies of the DNA repair protein XPA complexes with benzo[a]pyrene-DNA adducts" to be presented at the American Association for Cancer Research, New Orleans, March 24-28, 2001. This work also resulted in the submittal of several grant applications. The structural work on the BRCT domains is currently funded by NIH (M.Thelen, PI), the development of solid state NMR methods to study membrane proteins such as MOG is currently funded by a LW-LDRD (R. Maxwell, PI), and the development of single molecule techniques to study protein/DNA complexes is currently funded by a LW-LDRD (A. Noy, PI). The PI on this proposal has been invited to present seminars on the subject of MOG and damaged DNA/repair protein complexes at the Chemistry Departments at North Dakota State University, Fargo, ND (December 9, 1999) and University of North Dakota, Grand Forks, ND (December 10, 1999).

To overcome the second shortcoming of ${ }^{31} \mathrm{P}-\mathrm{NMR}$, sensitivity of detection, we designed and tested-in collaboration with the University of California, Davis (UCD) and LLNL Engineering Program — several novel RF microcoils. These microcoils increase the effective sample-to-coil ratio, and thereby increase the sensitivity of signal detection. Two abstracts on this work will be presented at the $42^{\text {nd }}$ Experimental NMR Conference, Orlando, FL, March 11-16, 2001. A record of invention (case no: IL-10775) has been filed for a new type of microprobe cassette design that integrates these microcoils together with the RF circuitry and sample chamber to enable easier handling and storage of rare, less than microliter volume samples. An UC Davis engineering graduate student, Kimball Yan, has received a LLNL graduate student employee fellowship to carry out and finish his Ph.D. thesis work at LLNL to design and build microfabricated nanoprobes specific for phosphorus NMR. The PI has been invited to present seminars on microcoil and nanoprobe developments at the Physics Department, University of Florida, Gainsville 
$\mathrm{Fl}$ (October 7,1999) and at the Department of Chemical Engineering, UC Berkeley, CA (September 28, 1999).

To overcome the third shortcoming of ${ }^{31} \mathrm{P}$, fast-relaxation, we used our DNA adducts and protein samples to develop and test new pulse sequences. Three publications that resulted from this work include: Journal of Biomolecular NMR, 12:177-182, 1998; Chemical Physics Letters, 302: 317-323, 1999; and Magn. Reson. Chem. 38:789-794, 2000. One is a method to correlate the rotational correlation time with the solvent accessible surface area, the second is an improved experimental scheme for measuring self-diffusion coefficients of biomolecules and the third is a novel, relaxation-compensated method to measure sample/solvent proton exchange rates. A poster abstract, "A novel relaxation compensated experiment to measure protein exchange rates in biomolecules based on decorrelation of heteronuclear two-spin order" was presented at the $41^{\text {st }}$ Experimental NMR Conference, Asilomar, CA, April 9-14, 2000.

The LDRD allowed us the opportunity to carry out this exciting research for the past two years and initiate several fruitful collaborations with researchers in engineering and C\&MS, UC Davis and U. Florida. Work on the NMR nanoprobe is continuing through the LLNL graduate student employee; a NSF grant proposal is in preparation using the preliminary results we have obtained during the course of this project. Work is also still in progress to study protein/DNA interactions and new grant proposals have been recently submitted to DARPA, DOE and NSF. Two current LW-LDRDs are in progress to more fully characterize the membrane protein MOG and to study protein/DNA complexes. A list of the resulting publications and meeting abstracts supported by this grant follows:

\section{$\underline{\text { Publications }}$}

2001

UCRL-JC-(paper should be submitted within two weeks - will have UCRL \# asap)

Solution Structure and Backbone Dynamics of the Human DNA Ligase III $\alpha$ BRCT Domain

to be submitted to Biochemistry

V. V. Krishnan, Kevin H. Thornton, Michael P. Thelen and Monique Cosman

2001

UCRL-JC-142465

Solution Structure of the 2-amino-1-methyl-6-phenylimidazo[4,5-b]pyridine C8deoxyguanosine adduct in duplex DNA submitted, Proceedings, National Academy of Sciences

Karen Brown, Brian E. Hingerty, Elizabeth A. Guenther, V. V. Krishnan

Suse Broyde, Kenneth W. Turteltaub and Monique Cosman. 
UCRL-JC-140456

Expression, Purification and Biophysical Characterization of the BRCT Domain of human DNA Ligase III $\alpha$

in press, Protein Expression and Purification

Kevin H. Thornton, V. V. Krishnan, Mary G. West, Jennifer Popham, Melissa Ramirez,

Michael P. Thelen and Monique Cosman

2000

UCRL-JC-135754

A novel relaxation compensated method to measure amide proton exchange rates in biomolecules based on decorrelation of heteronuclear two-spin order

Magnetic Resonance

Vol. 38, P. 789-794

Krishnan, V. V., M. Cosman

1999

UCRL-JC-132333

An improved experimental scheme to measure self-diffusion coefficients of biomolecules with an advantageous use of radiation damping

Chemical Physics Let

Vol. 302, P. 317-323

Krishnan, V. V., K. H. Thornton, M. Cosman

1998

UCRL-JC-128581

An empirical relationship between rotational correlation time and solvent accessible surface area.

J. Biomolecular NMR, 12: 177-182

Krishnan, V.V. and Cosman, M.

\section{Abstracts}

See copies of the 9 abstracts attached. 
American Neurological Association Meeting, Seattle WA, Oct. 6-10, 1999

\section{Structural Plasticity of A Protein Antigen Implicated In The Immunopathogenesis of Multiple Sclerosis}

Claude P. Genain

Department of Neurology, University of California, 513 Parnassus Ave, San Francisco, CA 94143-0435.

Kevin H. Thornton, Viswanathan V. Krishnan, Michael F. Mesleh, Monique Cosman Biology \& Biotechnology Research Program, Lawrence Livermore National Laboratory, Livermore, CA 94550 .

Myelin oligodendrocyte glycoprotein (MOG) is a surface-exposed component of the myelin sheaths implicated as a target antigen in autoimmune demyelinating disorders. In the disease model experimental allergic encephalomyelitis, autoantibodies to MOG mediate the disintegration of compact myelin sheaths into a disorganized array of small vesicles opsonized by macrophages; these autoantibodies have also been identified in acutely demyelinating lesions of human multiple sclerosis (MS) (Genain et al., Nature Medicine, 1999). A recent study of MOG-transfected cell lines suggests that the MOG-specific demyelinating antibodies bind to conformational, discontinuous epitopes on the molecule (Brehm et al., J. Neuroimmunol., 1999). Here, we report a solvent-dependent, $\beta$ to $\alpha$ transition in the conformation of the extracellular domain of recombinant MOG, as evidenced by CD, NMR and calorimetry. These results show an intriguing analogy to a growing number of central nervous system proteins, such as prions and amyloids, for which conformational switches are suspected to result in neurodegenerative disease. Preliminary data on the binding capacity of demyelinating antibodies to the different conformers of MOG will be presented. These findings demonstrate for the first time, that myelin self-antigens may undergo conformational changes that could dramatically affect the outcome of autoimmune responses in MS and related disorders.

Acknowledgements: Work performed at UCSF is supported by the National Multiple Sclerosis Society. Work performed at LLNL is under the auspices of the US Department of Energy by the Lawrence Livermore National Laboratory under contract W-7405-ENG-48 and supported by the Lawrence Livermore National Laboratory LDRD Director's Initiative grants 96-DI-010 and 99-LW-031. 
$41^{\text {st }}$ Experimental Nuclear Magnetic Resonance Conference, Asilomar Conference Center, April 9-14, 2000

A Novel Relaxation Compensated Experiment to Measure Proton Exchange Rates in Biomolecules Based on Decorrelation of Heteronuclear Two-Spin Order

\section{V. Krishnan and Monique Cosman}

Biology \& Biotechnology Research Program, 7000 East Ave., L-452, Lawrence Livermore National Laboratory, Livermore, CA 94551

A new combined experiment and analysis method based on decorrelation of heteronuclear two-spin order is presented for measuring accurate rapid amide proton exchange rates $\left(\mathrm{k}_{\mathrm{ex}}{ }^{\mathrm{HH}}=\mathrm{k}_{\mathrm{ex}}\right)$ in ${ }^{15} \mathrm{~N}$ labeled biomolecules, such as proteins or nucleic acids, in water. The term 'decorrelation' is defined as the loss of the initial correlation between a labile biomolecule proton and its coupled nitrogen when they are separated by intermolecular chemical exchange with water. The NMR pulse sequence (Decorrelation Exchange SpectroscopY (DECOREXSY)) measures the decay of the heteronuclear twospin order terms with minimal interference effects from relaxation processes and solvent induced artifacts. The new analysis protocol based on backbone relaxation measurements is introduced to compensate for relaxation contributions to the exchange rates that are otherwise inseparable. This simple and straightforward scheme has several potential applications in protein folding and biomolecular recognition and binding studies, and fills the need for a sensitive experiment to measure absolute fast-amide proton exchange rates predominantly in the sub-millisecond time scale. This work was performed under the auspices of the U.S. Department of Energy by the Lawrence Livermore National Laboratory (LLNL) under contract W-7405-ENG-48 and supported by the LLNL LDRD Director's Initiative grant 99-LW-031. 
$41^{\text {st }}$ Experimental Nuclear Magnetic Resonance Conference, Asilomar Conference Center, April 9-14, 2000

Solution Structure of the Ligase III $\alpha$ BRCT domain.

Kevin H. Thornton, V. V. Krishnan and Monique Cosman

Biology \& Biotechnology Research Program, 7000 East Ave., L-452, Lawrence Livermore National Laboratory, Livermore, CA 94551

BRCT domains are believed to mediate protein-protein interactions in the cell and are a part of several proteins involved in DNA repair and cell cycle checkpoints. The name is derived from the BRCA breast cancer genes and mutations within these domains are considered important in the carcinogenesis process. Here, we present the 3D solution structure of the human ligase III $\alpha$ BRCT domain (L3BRCT) determined by using NOE derived distance restraints, J-coupling constant data and amide proton exchange data. A combination of ${ }^{15} \mathrm{~N}$ and ${ }^{13} \mathrm{C}$-edited and ${ }^{13} \mathrm{C}$-filtered three dimensional experiments carried out on the protein at $\mathrm{pH} 6.5$ and $15^{\circ} \mathrm{C}$ yielded proton, nitrogen and carbon chemical shifts for the majority of the 86 residues. The 3D structure of L3BRCT shows structural homology to the $\mathrm{x}$-ray structure of the C-terminal BRCT domain of the repair protein XRCC1 (Zhang, et al. (1998) EMBO J. 17, 6404-6411). The hydrophobic core consists of a $\beta$-sheet, comprised of four parallel $\beta$-strands, with two $\alpha$-helices packed against one face of the $\beta$-sheet. One of the two $\alpha$-helices present in the XRCC1 BRCT structure is absent in the structure of the L3BRCT. The L3BRCT is observed to be a symmetric dimer in solution as evidenced by both NOE data and self-diffusion coefficient measurements. This work was performed under the auspices of the U.S. Department of Energy by the Lawrence Livermore National Laboratory (LLNL) under contract W-7405-ENG-48 and supported by the LLNL LDRD Director's Initiative grant 99-LW-031. 
$41^{\text {st }}$ Experimental Nuclear Magnetic Resonance Conference, Asilomar Conference Center, April 9-14, 2000

Chemical Shift Perturbation Studies of the DNA Repair Protein XPA Complexed with Benzo[a]pyrene-DNA Adducts

Monique Cosman and Viswanathan Krishnan, and Kevin Thornton

${ }^{1}$ Biology \& Biotechnology Research Program, 7000 East Ave., L-452, Lawrence Livermore National Laboratory, Livermore CA 94551

Benzo $[a]$ pyrene (BP) is a ubiquitous environmental pollutant that is metabolized in the cell to highly reactive diol epoxide (BPDE) intermediates that can covalently modify DNA. These DNA adducts exemplify a wide range of alterations in DNA structure and dynamics likely to be encountered by repair proteins and polymerases. More significantly, the biological activities of the compounds are dependent on the stereochemistry of the parent BPDE and of the reaction products. For example, the $(+)-$ $\mathrm{BP}$ diol epoxide is carcinogenic, while the (-)-enantiomer is not. Thus, it is important to determine how the adducts derived from the different parent isomers having opposite biological activities will interact with a damage recognition protein. Here, we report the ${ }^{1} \mathrm{H}$ and ${ }^{15} \mathrm{~N}$-chemical shift perturbation studies of the minimal binding domain of XPA (mbdXPA), a DNA damage binding protein in the nucleotide excision repair pathway with the unmodified control and two stereomeric $(+)$ - and (-)-trans-anti-benzo[a]pyrene$\mathrm{N}^{2}$-guanine ([BP]dG) adduct duplexes. Small but very distinct proton and nitrogen shifts are observed in the HSQC spectra of ${ }^{15} \mathrm{~N}$-labeled mbdXPA upon addition of each of the 3 DNA substrates, suggesting that the protein-DNA interactions are different in the absence of damage or in the presence of a carcinogenic versus noncarcinogenic lesion. These differences have been mapped onto the solution structures of the mbdXPA and may help explain why XPA promiscuously binds to both undamaged DNA and many different types of damaged DNA substrates, but with differing biological endpoints. That is, undamaged DNA is not repaired and the different adducts are repaired with varying efficiency. This work was performed under the auspices of the U.S. Department of Energy by the Lawrence Livermore National Laboratory (LLNL) under contract W-7405ENG-48 and supported by the LLNL LDRD Director's Initiative grant 99-LW-031. 
Poster Discussion Presentation:

American Association for Cancer Research, New Orleans, LA March 24-28' 2001

Biophysical Characterization Studies of the DNA Repair Protein XPA Complexed with Benzo[a]pyrene-DNA Adducts

Monique Cosman, Michele Corzett, Kathy M Severyn, Kevin H. Thornton, Viswanathan V. Krishnan, and Rod Balhorn

Biology \& Biotechnology Research Program, 7000 East Ave., L-448, Lawrence Livermore National Laboratory, Livermore CA 94551

Benzo[a]pyrene (BP) is a ubiquitous environmental pollutant that is metabolized in the cell to highly reactive diol epoxide (BPDE) intermediates that can covalently modify DNA. These DNA adducts exemplify a wide range of alterations in DNA structure and dynamics likely to be encountered by repair proteins and polymerases. More significantly, the biological activities of the compounds are dependent on the stereochemistry of the parent BPDE and of the reaction products. For example, the $(+)$ BP diol epoxide is carcinogenic, while the (-)-enantiomer is not. Thus, it is important to determine how the adducts derived from the different parent isomers having opposite biological activities will interact with a damage recognition protein. Fluorescence and thermodynamic studies of XPA, a DNA damage binding protein in the nucleotide excision repair pathway, with the unmodified control and the four stereomeric $(+)$ - and (-)-trans- and cis-anti-benzo[a]pyrene- $\mathrm{N}^{2}$-guanine ([BP]dG) 59 mer adduct duplexes show that the interactions are highly dependent on the stereochemistry of the BP ligand. In addition, the ${ }^{1} \mathrm{H}$ and ${ }^{15} \mathrm{~N}$-chemical shift perturbation NMR studies of the minimal binding domain of XPA (mbdXPA), with the unmodified control and two stereomeric $(+)$ - and (-)-trans-anti-benzo $[a]$ pyrene- $\mathrm{N}^{2}$-guanine $([\mathrm{BP}] \mathrm{dG}) 11$ mer adduct duplexes are also reported. Small but very distinct proton and nitrogen shifts are observed in the HSQC spectra of ${ }^{15} \mathrm{~N}$-labeled mbdXPA upon addition of each of the 3 DNA substrates, suggesting that XPA interacts differently with DNA when it binds to undamaged DNA or DNA containing carcinogenic versus noncarcinogenic lesions. These differences have been mapped onto the solution structures of the mbdXPA and are being used to determine why XPA promiscuously binds to both undamaged DNA and many different types of damaged DNA substrates, but with differing biological endpoints. Undamaged DNA is not repaired, while different adducts are repaired with varying efficiency. This work was performed under the auspices of the U.S. Department of Energy by the Lawrence Livermore National Laboratory (LLNL) under contract W-7405-ENG-48 and supported by the LLNL LDRD Director's Initiative grant 99-LW-031. 
Poster Presentation:

American Association for Cancer Research, New Orleans, LA March 24-28' 2001

Solution Structure of the 2-amino-1-methyl-6-phenylimidazo[4,5-b]pyridine (PhIP)-C8$\mathrm{dG}$ Adduct Opposite $\mathrm{dC}$ in a DNA Duplex

Karen Brown ${ }^{1}$, Elizabeth Guenther ${ }^{1}$, Brian E. Hingerty ${ }^{2}$, Suse Broyde ${ }^{3}$, Ken Turteltaub ${ }^{1}$, Monique Cosman ${ }^{1}$

${ }^{1}$ Biology \& Biotechnology Research Program, L-448, Lawrence Livermore National Laboratory, Livermore CA 94551; ${ }^{2}$ Life Sciences Division, MS-6480, Oak Ridge National Laboratory, Oak Ridge, TN 37830; ${ }^{3}$ Biology Dept, New York University, New York, NY 10003

The heterocyclic amine PhIP is a mutagenic compound that is formed during the cooking of fish and meats and has been shown to pose a significant dietary cancer risk to humans. We have used a combined NMR-molecular mechanics computational approach to determine the solution structure of the major C8-dG adduct formed by reaction of $\mathrm{N}$ acetoxy-PhIP with an 11 mer duplex sequence. The NMR data show that the [PhIP]dG.dC 11 mer duplex undergoes a slow conformational exchange in which the PhIP ligand either intercalates between the flanking $\mathrm{dG} \cdot \mathrm{dC}$ base pairs by displacing the modified $\mathrm{dG}$ residue into the major groove, or is located outside the helix and solvent exposed in a minimally perturbed B-DNA duplex. The amount of the external binding conformer present is temperature dependent and ranges from $\sim 10 \%$ at $25^{\circ} \mathrm{C}, 15 \%$ at $20^{\circ} \mathrm{C}$ and $32 \%$ at $1{ }^{\circ} \mathrm{C}$ The PhIP phenyl ring undergoes an additional fast rotation with respect to the imidazopyridine fused ring system. However, the phenyl ring is clearly forming stacking interactions with the deoxyribose sugar protons of the flanking $\mathrm{dG}$ residues on the complementary strand in the main base-displaced intercalation structure, as evidenced by the unusually shifted proton resonances of these sugar protons due to the phenyl ring current effects contributions. Both proton and carbon chemical shift data for the sugar resonances of the PhIP modified dG are consistent with a syn glycosidic torsion angle for this residue, which is directed toward the 3 'end of the modified strand. The conformational equilibrium observed for the PhIP-C8-dG adduct may, in part, explain the variety of the types of mutations induced by this adduct. This work was carried out under the auspices of the U.S. DOE by LLNL (W-7405-ENG-48), by ORNL

(DEAC0500OR22725) and supported by LLNL LDRD Director's Initiative grant 99-LW031 and NIH CA55861. 
ACS meterig, hew Orleans, Dec, zove

XCRL- $T C-141178$ ABS

INVESTIGATION OF THE SECONDARY STRUCTURAL CHANGES IN RMOG[1-125] BY

CIRCULAR DICHROISM. M. Ngu-Schwemlein*, M. Corzette, R. Balhorn and M. Cosman, *Department of Chemistry, Southern University, Baton Rouge, LA, 70813, Biology and

Biotechnology Research Program, Lawrence Livermore National Laboratory, Livermore, CA 94551

Myelin oligodendrocyte glycoprotein (MOG) has recently been implicated as a prime autoantigen leading to autoimmune demyelination in animal models. In this study, the expression of the soluble form of non-glycosylated extracellular domain of this glycoprotein, rMOG[1-125], rMOG[1-125] was obtained in an E. Coli M15(pRep4) host. The conformational change and stability of rMOG[1-125] at physiological $\mathrm{pH}$ has been followed using Circular Dichroism. The interaction of rMOG[1-125] with trifluorethanol, sodium lauryl sulfate, neutral detergents, dodecylphosphocholine (DPC) and palmitoyllyso-phosphocholine (LPCP), an anionic detergent, palmitoyllysophosphatidic acid (LPAP) and mixtures of these detergents will be presented. This work was carried out under the auspices of the U.S. DOE bv LLNL (W-7405-ENG-48).

ABSTRACT. Please be BRIEF-150 words maximum if possible. Title of paper should be ALL CAPS; author(s) listed by first name, middle initial, last name; indicate address with zip code.
A. DIVISION OF BIOL

\section{B. ACS MEMBER}
$\bigotimes$ Yes
Division Member
$\square$ Yes
No
C. TITLE OF PAPER
Investigation of the Secondary Structural
Changes in rMOG[1-125] by Circular Dichroism

Please indicate preference: $\square$ Oral $\square$ Poster

$\square$ withdraw if oral/poster format cannot be met

\section{AUTHORS}

Principal Author:

Last Name

First Name

M.

Ngu-Schwemlein

Presenting Author (if different):

Co-authors:

$\begin{array}{ll}\text { Corzette } & \mathrm{M} . \\ \text { Balhorn } & \mathrm{R} . \\ \text { Cosman } & \mathrm{M} .\end{array}$

Cosman M
( To be filled in by Division)

Paper number as listed on program

E. Principal Author's Business Mailing Address Including Zip Code

Department of Chemistry Lee Hall Rm. 108

Southern University

Baton Rouge, LA 70813

F. Principal Author's Telephone, Fax Number, and E-mail Address

Phone: (225) 771-3734

FAX: (225) 771-3992

E-mail: mariamin@aol.com

G. For contributed papers, do authors meet criteria outline in ACS Bylaw VI, Section 6(3)? See instructions.

$\bigotimes$ Yes $\square$ No

NOTE: ALL PRESENTING AUTHORS MUST REGISTER FOR THE MEETING-EITHER FULL MEETING REGISTRATION OR ONE-DAY REGISTRATION FOR THE DAY OF PRESENTATION.

H. Specify Equipment Required for Presentation Other than 2" $\times 2$ " (35 MM) slide or overhead (transparency) projector. There may be a charge to the individual or division for equipment. Please check with Program Chair.

Equipment 


\section{Submitrex to TID \\ $\times / x \in \cos$}

$42^{\text {st }}$ Experimental Nuclear Magnetic Resonance Conference, Orlando, FL March 11-16 2001

Practical and repeatable manufacturing of NMR micro-coils using the Laser-Lathe method.

Vince Malba, Leland B. Evans, Monique Cosman, Kin Yan, Robert Maxwell

Lawrence Livermore National Laboratory, Livermore CA 94551

It is well established that sub-millimeter RF coils can provide a valuable increase in sensitivity for mass limited samples. Processes for the repetitive and versatile manufacture of such coils to date, however, have been limited. We have developed a new simple and repeatable method for the fabrication of NMR microcoils on curved glass substrates such as capillaries. The process utilizes a laser direct-write system consisting of a focused Ar-ion laser, a spindle stage for rotation and a linear stage for translation of the target. A positive electro-deposited photoresist is exposed by the Laser-lathe system and copper is electroplated through the resist mask to form the RF coil. We show that the Laser-lathe process is easily variable for different diameter tubes, different coil geometries (saddle and solenoid, for example), and different pitches and numbers of turns. Comparison of actual and theoretical properties of L-lathe generated coils to handwound coils are favorable. This work was performed under the auspices of the U.S. Department of Energy by University of California Lawrence Livermore National Laboratory under contract No. W-7405-Eng-48 and supported by the LLNL LDRD Director's Initiative grant 99-LW-031. 


$$
\begin{aligned}
& \text { Labmither to THD } \\
& \text { र/xb/oi }
\end{aligned}
$$

$42^{\text {st }}$ Experimental Nuclear Magnetic Resonance Conference, Orlando, FL March 11-16 2001

Design, Analysis and Fabrication of Microfabricated Multi-turn Helmholtz Microcoils for High Resolution NMR Applications.

Kin Yan *\#, V.V.Krishnan*, Monique Cosman*, Jeff de Ropp\#, Jeff Walton\#, Rosemary Smith\#, and Scott Collins\#

*Lawrence Livermore National Laboratory, Livermore CA 94551

\#University of California, Davis, CA, 95616

Recently, microcoil design for NMR spectroscopy has received a renewed interest (1) due to their potential capability to analyze mass limited samples in micro to nano liter volumes and their applications in high throughput screening methods (2). The signal to noise ratio of NMR experiments can be increased by optimizing the coil sensitivity (3). One way to accomplish this goal is by decreasing the coil dimensions. In this work we present the design, numerical analysis, fabrication and testing of a multi-turn Helmholtz microcoil for high-resolution NMR applications. The probe head is designed to have two multi-turn spiral surface coils fabricated on a Pyrex substrate using microfabrication technology. It also contains a spherical sample chamber to minimize static field distortions and capillary channels for easier sample handling. Numerical simulations were performed using MATLAB based on a simplified surface coil model. The design parameters are optimized for detecting ${ }^{31} \mathrm{P}$ nucleus for a maximum signal to noise at 242 $\mathrm{MHz}$ (14.1 T, $600 \mathrm{MHz}$ proton). The probe head is mounted on a printed circuit board and micro strip lines are used to minimize the lead inductance. Preliminary NMR results and the advantages and associated problems in using Helmhotlz micro-coils will also be presented. This work was performed under the auspices of the U.S. Department of Energy by the Lawrence Livermore National Laboratory (LLNL) under contract W-7405ENG-48 and supported by the LLNL LDRD Director's Initiative grant 99-LW-031.

1. A G Webb, Progress in Nuclear Magnetic Resonance Spectroscopy, 31, 1-42, (1997).

2. Yi. Li et al., Analytical Chemistry, 71, 4815-20, (1999).

3. D I Hoult, R E Richards, Journal of Magnetic Resonance, 24, 71-85, (1976). 